\title{
Escrita académica e plágio no ensino superior
}

\author{
Isabel Festas \\ Armanda Matos \\ Ana Seixas \\ Universidade de Coimbra (Portugal)
}

\section{Resumo}

O plágio pode ser visto como um problema de literacia, relacionado com dificuldades na escrita académica. $\bigcirc$ principal objetivo deste estudo foi construir e validar um questionário destinado a avaliar as percepções de estudantes universitários acerca das suas competências de consulta, citação, compreensão e escrita a partir de fontes. O estudo incluiu 202 estudantes. Foram feitas análises fatoriais através de análises de componentes principais, tendo-se encontrado: três fatores para a escala estratégias de compreensão (seleção, organização, integração); três para a escala estratégias de escrita (planificação, revisão e "penso-logo-escrevo"); três para a escala estratégias de escrita a partir de fontes (cópia, mosaico/paráfrase, sumário); dois para a escala estratégias de consulta (elementares, elaboradas). A escala conhecimento das normas de citação revelou uma boa fidelidade. O questionário desenvolvido é essencial para futuramente se estudarem as relações entre o plágio e as competências de literacia.

Palavras-chave: Plágio. Escrita académica. Literacia. Ensino superior

\section{Academic writing and plagiarism}

\section{Abstract}

Plagiarism can be seen as a literacy academic matter related to academic writing difficulties. The main goal of the current study was to develop and validate a questionnaire that would measure the perception of college students about their consulting, citation, comprehension, and writing from sources competencies. The study included 202 undergraduate students. Exploratory factor analyses were conducted using principal components analysis, having being found: three factors for comprehension strategies scale (selection, organization and integration); three factors for writing strategies scale (planning, revision, and "think-then-do"); three for writing from sources strategies scale (copy, patchwriting/paraphrase, and summary); two for consulting strategies scale (elementary, elaborated). The citation norms knowledge scale showed a good fidelity. The questionnaire developed will be essential in future research aiming to study the relationship between plagiarism and literacies competencies.

Keywords: Plagiarism. Academic writing. Literacy. High education. 


\section{Escritura académica y plagio en la educación superior}

\section{Resumen}

El plagio puede verse como un problema de alfabetización, relacionado con dificultades en la redacción académica. El objetivo principal de este estudio fue construir y validar un cuestionario diseñado para evaluar las percepciones de los estudiantes universitarios sobre sus habilidades de consulta, cita, comprensión y escritura a partir de fuentes. El estudio incluyó 202 estudiantes. Los análisis factoriales se realizaron a través del análisis de componentes principales, y se encontraron: tres factores para la escala de estrategias de comprensión (selección, organización, integración); tres para estrategias de escritura (planificación, revisión, "pensar-escribir-pronto"); tres para estrategias de escritura desde las fuentes (copia, mosaico/ paráfrasis, resumen); dos para estrategias de consulta (primaria, elaborada). La escala conocimiento de los estándares de citas reveló buena fidelidad. El cuestionario desarrollado es esencial para seguir estudiando la relación entre plagio y habilidades de alfabetización.

Palabra-claves: Plagio. Escritura académica. Alfabetización. Enseñanza superior.

\section{Introdução}

O plágio e a integridade académica são considerados atualmente questões cruciais em todos os níveis de ensino, com particular destaque para o superior (BRETAG, 2016). Reconhecendo a importância destes temas, o seu estudo tem sido feito sobretudo numa perspetiva ética e moral, salientando-se a necessidade de conhecer os comportamentos e motivações de quem plagia (EAST, 2010) e, consequentemente, de implementar a vigilância e os meios de deteção do plágio (WEBER-WULFF, 2014). No entanto, nas últimas décadas, alguns autores têm chamado a atenção para a necessidade de situar este problema no âmbito da literacia académica, destacando as fragilidades dos estudantes na consulta, escolha e citação das fontes, bem como as suas dificuldades de compreensão e de escrita, apelando, em simultâneo, à necessidade de se desenvolverem medidas pedagógicas em alternativa às mais usuais de natureza punitiva (HOWARD; ROBILLARD, 2008; PECORARI, 2016).

A escrita académica, envolvendo conhecimentos e competências de natureza diversificada, requer, efetivamente, um bom nível de literacia, cuja ausência pode ser responsável por uma produção escrita deficitária, facilmente identificada como plágio. Textos muito próximos dos originais podem não corresponder a uma fraude, mas resultar da incapacidade dos seus autores para 
procurar, usar e compreender as fontes e para escrever de modo autónomo, respeitando as regras de citação académicas.

Podemos considerar que as dificuldades na escrita de textos académicos começam logo na procura das fontes. Com efeito, escrever implica uma escolha criteriosa das fontes a consultar, tarefa nem sempre fácil, sobretudo se pensarmos que, na atualidade, esta realidade se reporta, em grande parte dos casos, ao mundo digital e da Internet. Relativamente a este aspeto, mais importante do que afirmar, como fazem alguns autores, que estes últimos são meios facilitadores do plágio (BENNETT, 2005), será realçar a ausência de uma literacia digital e mediática, responsável por uma consulta que não obedece a critérios rigorosos (BUCKINGHAM, 2007).

A procura das fontes requer uma compreensão das especificidades da literatura especializada relativamente à de divulgação. Nem sempre os estudantes têm a noção destas diferenças, verificando-se, no caso particular da Internet, que muitas vezes as buscas são feitas nos mesmos sites em que se procura a informação usada no dia a dia, e não em fontes mais sofisticadas e adequadas aos fins académicos (JOINT INFORMATION SYSTEMS COMMITTEE AND THE BRITISH LIBRARY, 2012). Uma avaliação das fontes baseada no 3 conhecimento da sua origem é fundamental para perceber que sites como o Google, por exemplo, têm interesses comerciais que ditam a informação que está disponível, enquanto os sites académicos e de comunidades científicas disponibilizam estudos desenvolvidos e validados pelos especialistas das áreas disciplinares em que o estudante se deve integrar (DAVIES; HOWARD, 2016). Podendo esta dificuldade ao nível da procura e da seleção das fontes adequadas não ser diretamente responsável pela escrita de textos "colados" do original, ela é, no entanto, reveladora da iliteracia que parece caracterizar muitos dos estudantes do ensino superior.

Os problemas em citar corretamente, denotando, também, uma falta de literacia, constituem um fator que pode contribuir mais diretamente para a redação de textos classificados como plágio. A existência de normas específicas de diferentes áreas académicas, com convenções e estilos próprios, aumenta o grau de exigência requerido na citação correta e apropriada. De acordo com cada sistema de regras, o estudante deve reconhecer o que retirou das fontes, pondo aspas quando necessário, citando e atribuindo as ideias aos autores originais. Muitos estudantes manifestam problemas no conhecimento destas regras, reconhecendo-os como uma das causas de escreverem 
textos suscetíveis de serem considerados como tendo sido plagiados (GULLFER; TYSON, 2010).

Escrever a partir de fontes exige, igualmente, competências de compreensão e de composição que é pressuposto terem-se desenvolvido ao longo de toda a escolaridade. A produção de um trabalho escrito é um processo complexo que envolve a construção de textos bem organizados e que integrem as informações e os conhecimentos elaborados através da consulta e da leitura de muitas fontes, em papel ou online. Para tal, é necessário compreender os documentos estudados, o que passa pela seleção da informação importante, pela sua organização e pela sua integração nos conhecimentos anteriores (VAN DIJK; KINTSCH, 1983). Tratando-se de escrita académica, as informações reportam-se a domínios muito especializados, o que aumenta o grau de dificuldade na construção do modelo do texto, necessário a uma compreensão completa e condição indispensável a uma verdadeira aprendizagem (KINTSCH, 2009). Efetivamente, para que a leitura das fontes conduza a algo mais do que à sua simples reprodução, o leitor precisa de ligar o que lê e o que já sabe, dependendo a riqueza dos conhecimentos assim elaborados da possibilidade de criar novas relações. Só desse modo the é permitido escrever textos que não sejam decalcados dos originais. É preciso, ainda, considerar que a consulta e leitura de documentos múltiplos e diversificados implica que o leitor seja capaz de integrar a informação recolhida num todo coerente e ajustado a um determinado fim, exigência esta que vai além da que está envolvida no estudo de um único texto (ROUET, 2006).

A compreensão assim entendida, sendo condição necessária a uma escrita autónoma e independente das fontes, não é, no entanto, suficiente, já que esta última exige competências específicas e complexas. Ao respeito das regras ortográficas, de pontuação e sintáticas, junta-se o recurso adequado a estratégias de planificação, de composição e de revisão (HAYES; FLOWER, 1980). Podendo ser usadas de forma sequencial (primeiro planifica-se, a seguir compõe-se e, por último, revê-se) ou recursiva (por exemplo, vai-se revendo à medida que se compõe, ou vai-se planificando no decurso da escrita), tudo indica que a combinação destas estratégias pode variar em função do tipo de tarefa e também de escritor, havendo, no entanto, padrões de utilização mais eficientes do que outros (TORRANCE; THOMAS; ROBINSON, 2000).

Quando alguém domina as regras de citação e está habilitado para consultar e elaborar os conhecimentos das fontes, isto é, para os transformar 
através da integração nos conhecimentos anteriores, e de recorrer a combinações de estratégias adequadas, planificando, compondo e revendo de modo eficiente, torna-se capaz de escrever textos autónomos e originais (BEREITER; SCARDAMALIA, 1987; KELLOGG, 2008). No entanto, sabe-se que muitos estudantes de todos os níveis de ensino, incluindo os do superior, manifestam dificuldades significativas em escrever desta forma (GRAHAM; EARLY; WILCOX, 2014; IAVE, 2018; ODENA; BURGESS, 2017), recorrendo àquilo que Bereiter e Scardamalia (1987) chamam de evocação de conhecimentos, isto é, à pura transcrição do que se lembram ou do que leram. Sem elaborar/transformar as fontes, não as integrando nos conhecimentos prévios, sem planificar o que pretendem escrever, sem rever em função dos objetivos delineados, não é de admirar que muitos universitários produzam textos que, não se distinguindo dos orais, sejam, simultaneamente, muito próximos dos originais, confundindo-se facilmente com plágio.

Estudando a escrita de estudantes do ensino superior e particularmente dos que tinham o inglês como segunda língua, Pecorari (2008) constatou que, devido às suas dificuldades, e para não deturparem o sentido, eles usavam como estratégia uma certa "colagem" da linguagem expressa nos textos 5 consultados. Esta dependência da linguagem das fontes pode facilmente ser confundida com o plágio. Na linha deste trabalho, outros, sobretudo os que têm sido conduzidos no âmbito do Citation Project (HOWARD, 2016; JAMIESON, 2013, 2018; JAMIESON; HOWARD, 201 1, 2019), têm confirmado esta estratégia e alargado a compreensão da sua dimensão. De facto, tem-se verificado que os problemas de literacia conduzem à adoção de estratégias que vão da cópia pura e simples ao que alguns apelidaram de escrita em mosaico (patchwriting) (HOWARD, 1992).

Trata-se de uma forma de escrita em que os autores se prendem à linguagem do texto consultado, fazendo pequenas alterações para evitar a cópia. Supressões e substituições de algumas palavras por outras muito próximas, mudanças morfológicas (por exemplo, no tempo dos verbos) e de sintaxe (por exemplo, passar uma frase ativa para passiva) são características desta estratégia. Os autores vão reproduzindo o original, frase a frase, sem atender ao texto como um todo. Uma outra estratégia a que os estudantes recorrem é a paráfrase. Assentando numa escrita mais desligada da linguagem expressa no texto, com uso de novos vocábulos e alteração das expressões, e considerada mais adequada, sendo mesmo objeto de ensino em muitas Universidades 
(YOSHIMURA, 2015), a paráfrase continua a basear-se na frase e não no texto. sumário, estratégia mais elaborada, é raramente usado, contrariamente ao que seria expectável em relação a estudantes do ensino superior UAMIESON, 2018 ). Caracterizando-se por apresentar as ideias principais do texto, o sumário pressupõe, entre outros, um trabalho de organização, de integração, de planificação, de composição e de revisão, que permitiria a quem escreve autonomizar-se dos aspetos superficiais das fontes, redigindo produtos originais. Na ausência das competências necessárias a um tal trabalho, os estudantes produzem textos decalcados, muito próximos dos que foram consultados, confundindo-se, desse modo, com os que são plagiados intencionalmente.

É atendendo ao cima exposto que o plágio pode ser perspetivado como uma questão de literacia, mais do que como um problema moral e ético. Na verdade, em muitos casos, reporta-se a défices no tratamento das fontes, seja ao nível da consulta, seja da citação, e a problemas de compreensão e de composição escrita, não podendo ser considerado como uma fraude que o estudante queira cometer (HOWARD, 2016; PECORARI, 2008). Nesta perspetiva, uma compreensão do plágio passa pelo estudo das competências de consulta, leitura e escrita dos estudantes, sendo, igualmente, fundamental perceber como é que os mesmos se percepcionam como leitores e como escritores. Terão a noção das suas reais capacidades? Focando-se muitos trabalhos no modo como o estudante vê o plágio, numa conceção segundo a qual este último é uma questão de literacia, o interesse desloca-se para aquilo que cada um diz acerca das suas competências de leitor e de escritor.

É neste contexto que se situa o presente estudo que se reporta ao trabalho de construção e de validação de um instrumento vocacionado para pesquisar o modo como o estudante se avalia relativamente a competências de literacia consideradas necessárias a uma escrita académica autónoma e independente das fontes consultadas. A obtenção de um instrumento desta natureza constitui um passo fundamental para o estudo das relações entre literacia e plágio. Inserindo-se o presente estudo num projeto mais alargado que intenta perceber o plágio como um problema de literacia, duas questões centrais são a de saber se os estudantes do ensino superior escrevem de forma adequada a partir de fontes e, em simultâneo, a de compreender se eles têm a noção das suas dificuldades e capacidades. Ao permitir a avaliação do que estudante diz fazer ou ser capaz de fazer como leitor, como pesquisador e como escritor, o instrumento que agora se apresenta responde ao segundo aspeto enunciado. 
Ao mesmo tempo o confronto dos resultados obtidos neste instrumento com os textos escritos pelos mesmos estudantes possibilitará, em estudos próximos, perceber se as suas capacidades correspondem ao que eles dizem fazer ou saber fazer.

\section{Participantes}

A amostra é composta por 202 estudantes do ensino superior que frequentavam o $1^{\circ}$ ciclo do curso de Ciências da Educação numa universidade pública da região centro de Portugal, sendo 118 (91.6\%) do sexo feminino e $15(7.4 \%)$ do sexo masculino, distribuídos pelos três anos de escolaridade: 118 $(58.4 \%)$ no $1^{\circ}$ ano, $46(22.8 \%)$ no $2^{\circ}$ ano e $36(17.6 \%)$ no $3^{\circ}$ ano. Registaram-se 2 casos omissos nas variáveis sexo e ano de escolaridade. Os participantes apresentavam idades compreendidas entre os 18 e os 38 anos $(M=20.5$; DP $=2.31$.

\section{Instrumento, procedimento e análise}

Com o objetivo de ter um instrumento capaz de avaliar o modo como os estudantes percepcionam as suas competências de literacia, nomeadamente aquelas diretamente relacionadas com uma escrita independente da linguagem usada nas fontes, foi construído um questionário com cinco escalas: estratégias de compreensão; estratégias de escrita; estratégias de escrita a partir de fontes; consulta de fontes; e conhecimentos sobre normas de citação.

A escala estratégias de compreensão, com 12 itens, incidia em estratégias de seleção, destinadas a selecionar a informação mais importante das fontes (e.g., Quando faço/escrevo trabalhos, a partir de leituras de artigos, livros ou outras fontes, tiro apontamentos do que leio), de organização, necessárias à estruturação da informação expressa nos textos consultados (e.g., Quando faço/escrevo trabalhos, a partir de leituras de artigos, livros ou outras fontes, faço esquemas do que li) e de integração, i.e., aquelas que permitem o estabelecimento de relações entre o que se estuda e os conhecimentos anteriores (e.g., Quando faço/escrevo trabalhos, a partir de leituras de artigos, livros ou outras fontes, relaciono o que li com conhecimentos que já tinha). As estratégias de compreensão foram avaliadas numa escala de 1 (Nunca) a 5 (Com 
muita frequência). Os itens foram elaborados a partir de teorias de referência sobre a compreensão e estudo de textos (VAN DIJK; KINTSCH, 1983).

A escala de estratégias de escrita incluía 17 itens visando avaliar as estratégias de planificação (e.g., Quando faço/escrevo trabalhos, a partir de leituras de artigos, livros ou outras fontes, faço, por escrito, um plano detalhado do texto, antes de começar a escrever), de composição (e.g., Quando faço/ escrevo trabalhos, a partir de leituras de artigos, livros ou outras fontes, escrevo o texto de uma só vez, sem fazer alterações) e de revisão (e.g., Quando faço/ escrevo trabalhos, a partir de leituras de artigos, livros ou outras fontes, verifico se o texto que escrevi tem os conteúdos necessários). A escala variava de 1 (Nunca) a 5 (Com muita frequência). A escolha e redação dos itens seguiu de perto a literatura (KELLOG, 1986) e outros instrumentos usados na investigação feita na área da composição escrita (TORRANCE; THOMAS; ROBINSON, 2000).

modo como os estudantes dizem usar as estratégias de escrita a partir de fontes foi avaliado por uma escala com 16 itens incidindo na cópia [e.g., Quando faço/escrevo trabalhos, a partir da leituras de artigos, livros ou outras fontes, copio uma ou outra frase da(s) fonte(s)], na escrita em mosaico (e.g., Quando faço/escrevo trabalhos, a partir da leituras de artigos, livros ou outras fontes, mudo os tempos de alguns verbos), na paráfrase (e.g., Quando faço/escrevo trabalhos, a partir da leituras de artigos, livros ou outras fontes, uso algumas frases da(s) fonte(s), escrevendo-as por palavras minhas) e no sumário (e.g., Quando faço/escrevo trabalhos, a partir da leituras de artigos, livros ou outras fontes, procuro escrever um texto novo em que relacione o que li com o que já sabial. As estratégias de escrita a partir de fontes foram avaliadas numa escala de 1 (Nunca) a 5 (Com muita frequência), tendo os seus itens sido baseados em estudos como os de Pecorari (2008) e dos autores do Citation Project (HOWARD, 2016).

A escala consulta de fontes incluía nove itens que procuravam avaliar a forma como os estudantes afirmam pesquisar os materiais de estudo, recorrendo a estratégias menos adequadas [e.g., Quando faço/escrevo trabalhos, a partir de leituras de artigos, livros ou outras fontes, vou à internet e consulto as primeiras hiperligações (links) que aparecem], ou mais elaboradas (e.g., Quando faço/escrevo trabalhos, a partir de leituras de artigos, livros ou outras fontes, procuro revistas especializadas da área de estudo; vou à internet, procurando em sites credíveis). A forma como os estudantes afirmam pesquisar os 
materiais de estudo foi avaliada numa escala de 1 (Nunca) a 5 (Com muita frequêncial.

A última escala pretendia avaliar o que os estudantes dizem saber sobre normas de citação e incluía 13 itens (e.g., Quando escrevo textos/trabaIhos a partir da leitura de fontes, sei: como e onde devo pôr aspas; como citar as informações que retiro da internet e de fontes online). $\bigcirc$ que os estudantes dizem saber sobre normas de citação foi avaliado numa escala de 1 (Discordo totalmente) a 5 (Concordo totalmente).

$\bigcirc$ questionário foi preenchido em contexto de sala de aula, tendo-se respeitado todos os requisitos de natureza ética necessários a este tipo de investigação (os participantes foram devidamente informados dos objetivos e responderam de forma voluntária, podendo desistir a qualquer momento). O tempo de preenchimento foi aproximadamente de 20 minutos.

As análises realizadas tiveram como objetivo examinar as características psicométricas do questionário. A estrutura fatorial dos itens que compõem as diferentes escalas do questionário foi estudada recorrendo à análise fatorial exploratória (análise de componentes principais e rotação varimax). A consistência interna dos fatores identificados foi analisada mediante o cálculo do 9 coeficiente Alpha de Cronbach. As análises foram efetuadas recorrendo ao SPSS (versão 22, 2013).

\section{Resultados}

Previamente à realização da análise fatorial exploratória, foi analisada a presença e distribuição de casos omissos. Encontrou-se uma prevalência não superior a $1.5 \%$ em todos os itens das escalas que compõem o questionário e constatou-se, ainda, a aleatoriedade da sua distribuição, pelo que se procedeu à sua substituição, utilizando o método de imputação pela média de cada variável.

Os pressupostos da análise fatorial exploratória foram analisados mediante o cálculo do valor de KMO IKaiser-Leyer-Olkin Measure of Sampling Adequacy), que se revelou em todos as extrações $\geq$ a .7, e a realização do teste de esfericidade de Bartlett, com valores de $\mathrm{p}<.001$ em todas as análises, revelando, desta forma, a significância das matrizes de correlações (HAIR; ANDERSON; TATHAM; BLACK, 1998). Concluiu-se, assim, ser viável recorrer 
ao método de análise fatorial exploratória para estudar a estrutura fatorial das escalas que compõem o questionário.

\section{Estratégias de compreensão}

A análise de componentes principais (ACP) extraiu três fatores com eigenvalues $>1$ (4.92, 1.30, 1.08) que explicavam $60.79 \%$ da variância total. Todos os itens revelaram cargas salientes $(\lambda$ 's $>$.5) em pelo menos uma das componentes, e apenas o item 10 (Quando faço/escrevo trabalhos, a partir de leituras de artigos, livros ou outras fontes, procuro outras fontes para compreender melhor o que li) correlacionou significativamente com duas componentes (Cl e C2).

A C 1 inclui 5 itens e avalia as estratégias de integração (e.g., Quando faço/escrevo trabalhos, a partir de leituras de artigos, livros ou outras fontes, relaciono o que li com conhecimentos que já tinhal. Apesar de o item 10 estar mais saturado no C2, foi integrado no C1, uma vez que, em termos teóricos, se enquadra melhor neste fator. As respostas nesta componente são bastante consistentes ( $\alpha$ Cronbach =.81). A C2 comporta quatro itens, visando as estratégias de organização (e.g., Quando faço/escrevo trabalhos, a partir de leituras de artigos, livros ou outras fontes, faço esquemas do que li). A consistência interna, sendo mais baixa do que a anterior, é aceitável ( $\alpha$ Cronbach $=.72$ ). A C3 integra três itens e avalia as estratégias de seleção (e.g., Quando faço/escrevo trabalhos, a partir de leituras de artigos, livros ou outras fontes, tiro apontamentos do que leio). A consistência interna mantém-se aceitável ( $\alpha$ Cronbach $=.731$.

\section{Estratégias de escrita}

A ACP extraiu inicialmente quatro fatores, com eigenvalues $>1$ (4.70, $2.24,1.84,1.43$ ). Estas componentes explicaram $60.04 \%$ da variância total. Uma vez que os quatro fatores identificados não apresentavam coerência teórica, a ACP foi repetida mas forçando a extração de três componentes apenas. Estas componentes explicaram $51.60 \%$ da variância total. Analisando os resultados, verificou-se que um dos fatores incluía dois itens, o 10 e o 16, que, do ponto de vista conceptual, se revelavam incongruentes, pelo que se decidiu 
repetir a ACP forçada a três componentes, excluindo esses dois itens. Estas componentes explicaram 55.65\% da variância total. Todos os itens revelaram cargas salientes $(\lambda$ 's $>$.5) em pelo menos uma das componentes.

A C 1 inclui sete itens e avalia aspetos relativos à revisão (e.g., Quando faço/escrevo trabalhos, a partir de leituras de artigos, livros ou outras fontes, verifico se o texto que escrevi tem os conteúdos necessários). A fidelidade é boa ( $\alpha$ Cronbach $=.81)$. A C2 inclui cinco itens que visam as estratégias de planificação (e.g., Quando faço/escrevo trabalhos, a partir de leituras de artigos, livros ou outras fontes, faço, por escrito, um plano detalhado do texto, antes de começar a escrever). A consistência interna desta escala é aceitável ( $\alpha$ Cronbach $=.77)$. A C3 reúne três itens que parecem avaliar uma estratégia de "penso-logo-escrevo" (e.g., Quando faço/escrevo trabalhos, a partir de leituras de artigos, livros ou outras fontes, vou pensando nos conteúdos à medida que vou escrevendo). A consistência interna desta componente é baixa (a Cronbach $=.66)$. Uma análise das estatísticas dos itens incluídos nesta componente revela que todas as correlações item-total são positivas e iguais ou superiores a .3, pelo que a diminuição no valor do coeficiente alfa pode estar relacionada com o pequeno número de itens.

\section{Estratégias de escrita a partir de fontes}

Inicialmente a ACP extraiu quatro fatores, com eigenvalues $>1$ (3.35, $2.46,1.32,1.211$. Estas componentes explicaram 52.12\% da variância total. Uma vez que a estrutura fatorial obtida não se revelou coerente do ponto de vista teórico, a ACP foi repetida forçando a extração de três componentes. Estas componentes explicaram $44.57 \%$ da variância total. Todos os itens revelaram cargas salientes $(\lambda ' s>.5)$ em pelo menos uma das componentes, com exceção de dois itens com cargas $>$.3. Estes dois itens mantiveram-se por se revelarem com grande interesse do ponto de vista teórico.

A Cl inclui seis itens incidindo em estratégias de escrita em mosaico le.g., Quando faço/escrevo trabalhos, a partir da leituras de artigos, livros ou outras fontes, mudo os tempos de alguns verbos), e de paráfrase (e.g., Quando faço/escrevo trabalhos, a partir da leituras de artigos, livros ou outras fontes, uso algumas frases da(s) fonte(s), escrevendo-as por palavras minhas). A fidelidade desta componente é razoável ( $\alpha$ Cronbach $=.72$ ). A C2 reúne quatro itens que avaliam a estratégia de cópia [e.g., Quando faço/escrevo trabalhos, 
a partir da leituras de artigos, livros ou outras fontes, copio uma ou outra frase da(s) fonte(s)]. Sendo a consistência interna desta componente relativamente baixa ( $\alpha$ Cronbach $=.65$ ), as correlações item-total são todas positivas e iguais ou superiores a .4, com exceção do item 3 (com correlação .2). $\bigcirc$ item 3 foi mantido por se mostrar teoricamente relevante [Quando faço/escrevo trabaIhos, a partir da leituras de artigos, livros ou outras fontes, uso algumas palavras da(s) fonte(s)]. De referir, ainda, que a fidelidade da escala não se alterava substancialmente, se o item 3 fosse excluído ( $\alpha$ Cronbach $=.68$ ). A C3 comporta seis itens correspondendo a estratégias de sumário (e.g., Quando faço/ escrevo trabalhos, a partir da leituras de artigos, livros ou outras fontes, procuro escrever um texto novo em que relacione o que li com o que já sabia). A fidelidade das respostas nesta subescala é relativamente baixa ( $\alpha$ Cronbach $=.62$ ). No entanto, as correlações item-total são todas positivas, iguais ou superiores a .3, com exceção do item 4 (Quando faço/escrevo trabalhos, a partir da leituras de artigos, livros ou outras fontes, procuro sintetizar o(s) texto(s) que li, escrevendo um texto mais pequeno), (com correlação .2), mas que se manteve por ser teoricamente relevante e por a sua exclusão não alterar substancialmente a fidelidade da escala global ( $\alpha$ Cronbach $=.63$ ).

\section{Consulta de fontes}

Inicialmente a ACP extraiu três fatores, com eigenvalues $>1$ (2.59, $1.61,1.04)$. Estas componentes explicaram $58.24 \%$ da variância total. Uma vez que, conceptualmente, a estrutura fatorial obtida não se revelou coerente, a ACP foi repetida forçando a extração de dois componentes. Estas componentes explicaram $46.63 \%$ da variância total. Todos os itens revelaram cargas salientes $\left(\lambda^{\prime} s>\right.$.5) em pelo menos uma das componentes.

A C 1 inclui dois itens que traduzem uma estratégia de consulta pouco cuidada [e.g., Quando faço/escrevo trabalhos, a partir de leituras de artigos, livros ou outras fontes, vou à internet e consulto as primeiras hiperligações (links) que aparecem]. A fidelidade desta componente é de 64. Sendo a consistência baixa, as correlações item-total são todas positivas e iguais ou superiores a.4, pelo que o valor baixo do coeficiente alfa pode estar relacionada com o pequeno número de itens. A C2 reúne os restantes sete itens que incidem em estratégias mais ponderadas de consulta (e.g., Quando faço/escrevo trabalhos, a partir de leituras de artigos, livros ou outras fontes, vou à internet, 
procurando em sites crediveis). A consistência interna desta componente é aceitável $(\alpha$ Cronbach $=.71)$.

\section{Conhecimentos sobre normas de citação}

Pretendendo esta escala avaliar aquilo que os estudantes dizem conhecer acerca das normas de citação, vigentes no seu domínio de estudo (normas APA), procedemos à determinação da sua consistência interna. Esta revelou-se alta ( $\alpha$ Cronbach $=.89)$. As correlações entre cada item e o total são todas positivas e estatisticamente significativas $(p<.001)$, variando entre .474 e.703.

\section{Discussão dos resultados, limitações e conclusões}

objetivo fundamental do presente estudo foi o de construir e validar uma medida multifatorial de avaliação do modo como o estudante do ensino superior percepciona as suas competências de consulta, compreensão e escrita

13 de textos a partir de fontes. $O$ trabalho efetuado permitiv elaborar um instrumento que avalia as percepções dos estudantes acerca de aspetos essenciais da literacia académica, relacionados com a possibilidade de produzir textos autónomos e desligados das fontes, condição necessária a uma escrita sem plágio.

Quanto às estratégias de compreensão que o estudante diz usar, foram encontrados os três fatores previstos, relativos a estratégias de seleção, organização e integração. Os dados encontrados vão ao encontro da literatura e da investigação feita nesta área que indicam que a compreensão de textos envolve a seleção da informação, a sua organização, através da identificação das ideias principais e do estabelecimento de relações entre as diferentes partes, e a sua integração nos conhecimentos anteriores (KINTSCH, 2009).

Relativamente ao modo como o estudante diz usar as estratégias de escrita, foram identificados três fatores, dois deles coincidentes com o que tinha sido previsto, mas um outro diferente, confirmando-se, assim, só em parte o que tinha sido hipotetizado para a escala original. As estratégias de planificação e de revisão foram claramente comprovadas, surgindo uma terceira estratégia que não aglomerou os itens inicialmente agrupados na composição. O 
que emergiu da análise fatorial foi uma variável que parece associada a uma estratégia "penso-logo-escrevo" que reuniu três itens, dois deles inicialmente atribuídos à planificação (Quando faço/escrevo trabalhos, a partir de leituras de artigos, livros ou outras fontes, vou pensando nos conteúdos à medida que vou escrevendo; Quando faço/escrevo trabalhos, a partir de leituras de artigos, livros ou outras fontes, vou pensando na estrutura do texto, à medida que vou escrevendo) e um outro à composição (Quando faço/escrevo trabalhos, a partir de leituras de artigos, livros ou outras fontes, escrevo um texto inicial em que mudo pequenas coisas).

Os outros três itens agrupados inicialmente na composição, depois da análise fatorial, distribuíram-se pelos restantes dois fatores, tendo um deles sido incluído nas estratégias de revisão/Quando faço/escrevo trabalhos, a partir de leituras de artigos, livros ou outras fontes, escrevo o texto de uma só vez, sem fazer alterações) e os outros dois excluídos porque, do ponto de vista conceptual, não se justificou a sua inclusão na categoria em que se situaram, relativa às estratégias de planificação (Quando faço/escrevo trabalhos, a partir de leituras de artigos, livros ou outras fontes, reescrevo o texto várias vezes, antes da versão final; Quando faço/escrevo trabalhos, a partir de leituras de artigos, livros ou outras fontes, escrevo um texto inicial em que mudo bastantes coisas).

Uma análise cuidada dos itens inicialmente atribuidos às estratégias de composição mostra-nos que, de facto, é difícil separar estas estratégias das que estão envolvidas na planificação e na revisão. Estratégias como mudar pequenas coisas ou escrever sem fazer alterações remetem necessariamente para formas de planificar e de rever que dificilmente se podem isolar daquilo que é a composição. Quanto ao fator que emergiu da análise fatorial, o mesmo corresponde a uma estratégia identificada na literatura (TORRANCE; THOMAS; ROBINSON, 2000). A estratégia "penso-logo-escrevo" parece caracterizar um modo de escrever usado em textos sobre assuntos muito familiares e/ ou por um tipo de escritor que escreve habitualmente desta forma. Trata-se de uma escrita em que há pouca planificação, em que se vai pensando à medida que se vai escrevendo, resultando num texto composto de uma só vez, em que se muda pouca coisa. Os resultados encontrados no nosso estudo estão, assim, enquadrados na investigação existente neste domínio. No entanto, é aconselhável desenvolver novos trabalhos com uma amostra maior e mais diversificada, 
incluindo estudantes de várias áreas académicas, no sentido de se verificar se a estrutura fatorial se mantém.

Três fatores foram identificados nas estratégias de escrita a partir de fontes, um que juntou a escrita em mosaico e a paráfrase, outro relativo à cópia e o último respeitante ao sumário. Relativamente ao que era previsto, enquanto a cópia e o sumário se viram reconhecidos como independentes, o mesmo não aconteceu com a escrita em mosaico e com a paráfrase, cujos itens foram incluídos num único fator. Embora muitos autores separem estas duas estratégias (JAMIESON, 2013), como vimos em cima, ambas têm em comum o facto de se basearem na frase e não no texto como um todo. Trata-se de uma escrita que trabalha a frase, de uma forma mais superficial, no caso da escrita em mosaico (suprimindo e substituindo algumas palavras por outras muito próximas, fazendo mudanças morfológicas e de sintaxe), ou mais elaborada, como acontece na paráfrase (recorrendo a novos vocábulos e alterando algumas expressões). Mas, em qualquer dos casos, a escrita vai-se construindo a partir das frases consultadas e não das ideias principais do texto, como acontece no sumário (HOWARD; SERVISS; RODRIGUE, 2010). Podemos, deste modo, afirmar que os resultados encontrados têm suporte teórico. Reconhecemos, no entanto, 15 que, no futuro, é desejável fazer outros estudos com esta escala, de forma a permitir aprofundar o conhecimento acerca da sua organização fatorial.

Encontraram-se dois fatores na consulta de fontes, um relativo a estratégias pouco adequadas e outro revelando uma consulta com algum critério, confirmando o que era previsto. Apesar disso, é recomendável desenvolver futuramente novos estudos em que, nomeadamente, se procure alargar o número de itens do primeiro fator. A escala incidindo naquilo que o estudante diz saber sobre normas de citação mostrou ter uma boa consistência interna, revelando constituir um instrumento útil neste tipo de estudos.

questionário agora validado é um instrumento muito importante para o alargamento dos estudos sobre as relações entre a literacia relativa à escrita académica e o plágio. Admitindo que este último, em muitos casos, não corresponde a uma fraude mas a uma ausência de competências de consulta, de compreensão e de escrita de textos, torna-se fundamental perceber melhor em que medida grande parte daquilo que se inclui atualmente na fraude académica não resulta de uma iliteracia.

Recorrendo ao instrumento aqui apresentado, em futuros trabalhos, poder-se-á estudar de modo aprofundado de que forma é que o estudante se 
percepciona como leitor, pesquisador e escritor, isto é, como é que ele avalia a sua literacia académica, e como se posiciona relativamente a cada uma das dimensões do questionário. As percepções que os estudantes têm relativamente aos vários aspetos das suas competências de literacia são equivalentes, por exemplo, um estudante que avalia bem o uso que faz de estratégias num determinado domínio, como a compreensão, também considera que recorre a estratégias adequadas de escrita e de consulta? Ou será que o estudante refere diferentes competências de acordo com cada um dos domínios le.g., achar que tem competências de consulta, mas não de escrita)? Análises mais finas das múltiplas variáveis envolvidas em cada uma das dimensões permitirão elucidar, também, qual a relação entre aspetos particulares dos diversos fatores.

Os que afirmam usar estratégias adequadas de compreensão, também referem que planificam, reveem e sumariam o que escrevem? Como é que aqueles que se avaliam como alguém que escreve à medida que vai pensando se veem do ponto de vista das estratégias de compreensão, de consulta e de escrita a partir das fontes que usam? Haverá diferenças entre estes e aqueles que afirmam recorrer a estratégias de planificação e de revisão dos textos que escrevem? E entre os que afirmam sumariar o que leem e os que reconhecem usar estratégias de escrita em mosaico/paráfrase ou de cópia? Há toda uma série de questões importantes que podem ser exploradas e ver-se estudadas através do instrumento agora apresentado.

Mais fundamental, ainda, é perceber como é que as percepções dos estudantes se relacionam com os seus desempenhos. Em estudos futuros, através do confronto destas percepções com a forma como os mesmos estudantes escrevem, será possível entender se estes últimos têm uma noção clara das suas dificuldades e/ou potencialidades no que respeita à literacia académica. Poder-se-á ver, também, as relações entre as múltiplas variáveis avaliadas no instrumento agora apresentado e os desempenhos. Por exemplo, quem diz usar estratégias de cópia é quem efetivamente as usa? Quem afirma recorrer ao sumário escreve textos autónomos das fontes consultadas ou, pelo contrário, não se distancia de uma escrita muito colada ao que consulta? Estas e outras questões poderão ser estudadas e respondidas futuramente, através de investigações que, recorrendo ao presente questionário, confrontem os seus resultados com os textos/trabalhos escritos pelos estudantes. 
Por último, todos estes dados em conjunto constituem um passo decisivo para pensar em intervenções capazes de ajudar a resolver um problema que é atualmente considerado como muito significativo (BRETAG, 2016). Uma intervenção com vista a ajudar os estudantes a escreverem autonomamente sem se colarem às fontes consultadas passa certamente pelos dois níveis aqui identificados, isto é, pelo desenvolvimento de competências de consulta, leitura e escrita de textos académicos e também por um trabalho ao nível da forma como eles se veem como leitores, como pesquisadores e como escritores.

\section{Nota}

1 Trabalho realizado no âmbito do Projeto COMEDIG - Competências de literacia digital e mediática em Portugal (PTDC/CED-EDG/32560/2017), cofinanciado pelo COMPETE 2020, Portugal 2020 e União Europeia, através do FEDER e pela Fundação para a Ciência e Tecnologia I.P./MCTES através de fundos nacionais (PIDDAC).

\section{Referências}

17 BEREITER, Carl; SCARDAMALIA, Marlene. The psychology of written composition. Hillsdale, NJ: Lawrence Erlbaum, 1987.

BENNETT, Roger. Factors associated with student plagiarism in a post-1992 university. Assessment and Evaluation in Higher Education, Londres, v. 30, n. 2, p.137-162, apr. 2005.

BRETAG, Tracey (org.). The handbook of academic integrity. Singapore: Springer, 2016. BUCKINGHAM, David. Digital media literacies: rethinking media education in the age of the Internet. Research in Comparative and International Education, Oxford, v. 2, n. 1, p. 43-55, mar. 2007.

DAVIES, Laura;HOWARD, Rebecca. Plagiarism and the Internet: Fears, facts, and pedagogies. In BRETAG, Tracey (ed.). The handbook of academic integrity. Singapore: Springer, 2016.

EAST, Julianne. Judging plagiarism: a problem of morality and convention. Higher Education, Londres, v. 59, p. 69-83, mayo 2010.

DOI: https://doi.org/10.1007/s 10734-009-9234-9. 
GRAHAM, Steve; EARLY, Jessica; WILCOX, Kristen. Adolescent writing and writing instruction: Introduction to the special issue. Reading and Writing: an Interdisciplinary Journal, Dordrecht, v. 27, n. 6, p. 969-972, oct. 2014. DOl: https://doi.org/10.1007/ s 11 145-0 1 4-9497-0

GULLIFER, Judith; TYSON, Graham. Exploring university students' perceptions of plagiarism: a focus group study. Studies in Higher Education, v. 35, n. 4 p. 463-48 1, jun. 2010. DOI: https://doi.org/10.1080/03075070903096508

HAIR, Joseph; ANDERSON, Rolph; TATHAM, Ronald; BLACK, William. Multivariate data analysis. 5th ed. Upper Saddle River: Prentice Hall, 1998.

HAYES, John; FLOWER, Linda. Identifying the organisation of writing processes. In: GREGG, Lee; STEINBERG, Erwin (eds.). Cognitive processes in writing. N.J: Hillsdale/ Erlbaum, 1980.

HOWARD, Rebecca. A plagiarism pentimento. Journal of Teaching Writing, Indianapolis, v. 11 , n. 2, p. 233-245, jan. 1992.

HOWARD, Rebecca. Plagiarism in higher education: An academic literacies issue? Introduction. In: BRETAG, Tracey (ed.). The handbook of academic integrity. Singapore: Springer. 2016.

HOWARD, Rebecca; ROBILLARD, Amy (eds.). Pluralizing, plagiarism: Identities, contexts, pedagogies. New York: Heinemann-Boynton/Cook, 2008.

HOWARD, Rebecca; SERVISS, Tricia; RODRIGUE, Tanya. Writing from sources, writing from sentences. Writing \& Pedagogy, Lacey, v. 2, n. 2, p. 177-192, mayo. 2010.

IAVE. Relatório nacional 2016 e 2017. Provas de aferição. Ensino Básico. Lisboa: Instituto de Avaliação Educacional, 2018.

JAMIESON, Sandra (2013). Reading and engaging sources: what students' use of sources reveals about advanced reading skills across the disciplines. Disponível em: http://wac. colostate.edu/atd/reading/jamieson.cfm. Acesso em: 3 mar. 2019.

JAMIESON, Sandra. The evolution of the Citation Project. Developing a pilot study from local to translocal. In: SERVISS, Tricia; JAMIESON, Sandra (eds.). The evolution of the Citation Project: lessons learned from a multi-year, multi-study. Utah: State University Press, 2018.

JAMIESON, Sandra; HOWARD, Rebecca. Unraveling the citation trail. Project information literacy smart talk, n. 8. 2011 . Disponível em: http://projectinfolit.org/smart-talks/ item/ 1 10-sandra-jamieson-rebecca-moore-howard-smart-talk. Acesso em: 4 abr. 2019. 
JAMIESON, Sandra; HOWARD, Rebecca. Rethinking the relationship between plagiarism and academic integrity. International Journal of Technologies in Higher Education/Revue Internationale des Technologies en Pédagogie Universitaire, v. 16, n. 2, set. 2019.

DOI: https://doi.org/10.18162/ritpu-2019-v16n2-07.

JOINT INFORMATION SYSTEMS COMMITTEE AND THE BRITISH LIBRARY 12012, june 28). Research of tomorrow: The research behaviour of generation $Y$ doctoral students. Disponível em: https://pdfs.semanticscholar.org/6943/75c5eabd948a59e46d3ab 14a d57b9e163d86.pdf. Acesso em: 6 maio. 2019.

KELLOGG, Ronald. Writing method and productivity of science and engineering faculty. Research in Higher Education, Dordrecht, v. 25, n. 2, p. 147-163, jun. 1986.

KELLOGG, Ronald. Training writing skills: A cognitive developmental perspective. Journal of Writing Research, Antuérpia, v. 1, n. 1, p. 1-26, jun. 2008.

KINTSCH, Walter. Learning and constructivism. In: TOBIAS, Sigmund; DUFFY, Thomas (eds.). Constructivist instruction: success or failure? New York: Routledge, 2009.

ODENA, Oscar; BURGESS, Hilary. How doctoral students and graduates describe facilitating experiences and strategies for their thesis writing learning process: A qualitative approach. Studies in Higher Education, v. 42, n. 3, p. 572-590, jul. 2017. DOl: https://

19 doi.org/10.1080/03075079.2015.1063598

PECORARI, Diane. Academic writing and plagiarism. A linguist analysis. London: Continuum International Publishing Group, 2008.

PECORARI, Diane. Plagiarism, international students, and the second-language writer. In: BRETAG, Tracey (ed.). The handbook of academic integrity. Singapore: Springer, 2016.

ROUET, Jean-François. The skills of document use. From text comprehension to Web-based learning. New York: Routledge. 2006.

TORRANCE, Mark; THOMAS, Glyn; Robinson, Elizabeth. Individual differences in undergraduate essay-writing strategies: a longitudinal study. Higher Education, Londres, v. 39, n. 4 , p. $181-200$, jun. 2000.

VAN DIIK, Teun; KINTSCH, Walter. Strategies of discourse comprehension. New York: Academic Press. 1983.

WEBER-WULFF, Debora. False feathers: a perspective on academic plagiarism. Berlin: Springer. 2014. 
YOSHIMURA, Fumiko. Japanese University students' task representations of paraphrasing and their experience with it. Tohoku Gakuin University Review: essays and Studies in English Language and Literature, Sendai, v. 99, p. 1-21, mar. 2015.

Profa. Catedrática Isabel Festas Universidade de Coimbra (Portugal)

Faculdade de Psicologia e de Ciências da Educação Centro de Estudos Interdisciplinares do Século XX (CEIS2O)

Orcid id: https:/ / orcid.org/0000-0002-1720-1488

E-mail: ifestas@fpce.uc.pt

Profa. Auxiliar Armanda Matos Universidade de Coimbra (Portugal)

Faculdade de Psicologia e de Ciências da Educação Centro de Estudos Interdisciplinares do Século XX ICEIS2O

Orcid id: https: / / orcid.org/0000-0003-0802-5870

E-mail: armanda@fpce.uc.pt

Profa. Auxiliar Ana Seixas Universidade de Coimbra, (Portugal)

Faculdade de Psicologia e de Ciências da Educação Centro de Estudos Sociais (CES) Orcid id: https: / / orcid.org/0000-0003-3 177-5493 E-mail: anaseixas@fpce.uc.pt

Recebido 2 mar. 2020

Aceito 18 ago. 2020 